\title{
CHINESE DUAL FREQUENCY WATER VAPOR RADIOMETER FOR VLBI
}

\author{
Xu Pei-yuan \\ Shanghai University of Science and Technology \\ Shanghai, People's Republic of China
}

A Chinese VLBI Network Project is under construction in China. The first 25-m antenna and associated VLBI system have just been completed at the She-Shan Station of the Shanghai Observatory. Both geodetic and astrophysical VLBI programs will be undertaken with the Network. A dual frequency water vapor radiometer is being developed to determine the tropospheric path delay correction. This WVR has the following characteristics:

\begin{tabular}{|c|c|}
\hline Operating frequencies & 20.60 and $31.65 \mathrm{GHz}$ \\
\hline IF bandwidth & $50-500 \mathrm{MHz}$ \\
\hline Noise figure & $\leq 6 \mathrm{~dB}$ \\
\hline Integration time & Software selectable in steps of $1 \mathrm{sec}$ \\
\hline Antenna configuration & $\begin{array}{l}\text { Two mechanically coupled elevation steerable } 90 \\
\text { offset paraboloid mounted on an azimuth pedestal }\end{array}$ \\
\hline Antenna beamwidth & $2.4 \mathrm{deg}$ \\
\hline Beam efficiency & $90 \%$ for \pm 5 deg around beam center \\
\hline Pointing accuracy & $0.5 \mathrm{deg}$ azimuth; $0.1 \mathrm{deg}$ elevation \\
\hline Control \& data acquisition & Microcomputer IBM PC/XT \\
\hline Calibration & $\begin{array}{l}\text { Waveguide load at } 313 \mathrm{~K} \text {, noise diode, tipping } \\
\text { curves }\end{array}$ \\
\hline Reference stability & Waveguide load $0.05 \mathrm{~K} /$ hour, diode $0.2 \% /$ week \\
\hline $\begin{array}{l}\text { Antenna temperature } \\
\text { accuracy }\end{array}$ & $0.5-1.0 \mathrm{~K}$ \\
\hline
\end{tabular}

